\title{
SGLT1 and SGLT1 Inhibitors: A Role to Be Assessed in the Current Clinical Practice
}

\author{
Leonardo Spatola (D) Silvia Finazzi - Claudio Angelini - Marco Dauriz •
}

Salvatore Badalamenti

Received: October 23, 2017 / Published online: November 24, 2017

(C) The Author(s) 2017. This article is an open access publication

\section{ABSTRACT}

Diabetes is a complex disease of increasingly common occurrence worldwide. Attaining optimal glycemic control is the main challenge to prevent the development of diabetes-related complications and/or to stop their progression. In recent years, the pharmacologic toolkit for the treatment of diabetes has considerably expanded, thus paving the way to more pathophysiology-oriented therapies. For instance, the sodium-glucose cotransporters SGLT2 and SGLT1 have been in the spotlight because of better knowledge of their physiology and therapeutic potential. At present, whereas the SGLT2 inhibitors are widely applied in current clinical practice as an effective and well-tolerated treatment that increases the urinary excretion of glucose, less is known about the use

Enhanced Content To view enhanced content for this article go to http://www.medengine.com/Redeem/ 35FCF06060F5CEDF.

L. Spatola $(\bowtie) \cdot$ S. Finazzi · C. Angelini ·

S. Badalamenti

Division of Nephrology and Hemodialysis,

Humanitas Clinical and Research Center, Milan,

Italy

e-mail: Leonardo.spatola@humanitas.it

M. Dauriz

Division of Endocrinology, Diabetes and

Metabolism, Department of Medicine, University of

Verona School of Medicine and Hospital Trust of

Verona, Verona, Italy of SGLT1 inhibitors. SGLT1s are of primary importance in the small intestine, an organ that does not express SGLT2, while in the kidney they are expressed in the late renal proximal tubules, where it reabsorbs the glucose escaped from the upstream SGLT2. Hence, SGLT1-mediated glucose reabsorption in the kidney is increased when the tubular glucose load overwhelms the capacity of SGLT2 or when the latter is inhibited. The role of SGLT1 in intestinal and renal glucose transport makes the transporter a potential target for antidiabetic therapy. Here, we briefly report the evidence on LX2761, a new inhibitor against SGLT1 and SGLT2 in vitro, which acts in vivo as a selective inhibitor of SGLT1 in the gastrointestinal tract. LX2761 improves glycemic control without the glycosuria-related side effects of SGLT2 inhibitors, particularly genitourinary tract infections. However, whether it represents a valid therapeutic option for all patients with diabetes or is more appropriate for specific phenotypes, e.g., patients with concomitant diabetes and chronic kidney disease, who may benefit less from the renal mechanism of selective SGLT2 inhibitors, remains to be tested in large randomized controlled trials.

Keywords: Glucose transporters; Glycemic control; SGLT1; SGLT1 inhibitors; SGLT2 


\section{COMMENTARY}

In recent years, the renal sodium/glucose cotransporters SGLT1 and SGLT2 have gained increasing attention from researchers and clinicians, given their potential role as an effective and generally well-tolerated treatment option for the attainment of a proper glycemic control $[1,2]$. However, the renal expression of both SGLT2 and SGLT1 in the proximal tubule of patients with type 2 diabetes (T2D) is still not completely elucidated, and experimental studies from different research groups have reported conflicting results [3-7].

In this regard, Solini et al. [3] recently reported that the renal expression of SGLT1 in patients with T2D was similar to that of their normoglycemic (NG) counterparts, which also showed higher expression of SGLT2. The patients included in the analysis had a mean diabetes duration of about 3 years and were on a glucose-lowering therapy with oral hypoglycemic agents, without SLGT2 inhibitors and/ or insulin therapy. These observations have not been reported by previous studies in mouse models of diabetes [4] or in human T2D patients [5-7]. As a potential explanation for their findings, the authors found that clinical factors such as duration of diabetes, different pharmacologic treatments, varying degrees of glycemic control and the extent of renal impairment may limit the comparability of the study findings and may have influenced the expression of each pair of brush border/basolateral transporters SGLT2/GLUT2 and SGLT1/GLUT1 located, respectively, in the S1-S2 and S3 segments of the tubular nephron.

However, Norton et al. [7] observed that in patients with T2D the expression of SGLT1 mRNA was increased more than fourfold in kidney biopsy specimens compared to individuals with normal glucose regulation. In this case, the authors argued that poorer glycemic control, older age and a decreased glomerular filtration rate may explain, at least in part, the increased expression of SGLT1 mRNA in patients with T2D. The authors also observed significant correlations of higher expression of SGLT1 mRNA with increasing levels of fasting plasma glucose $(r=0.63, \quad p<0.001)$, post-prandial glucose $(r=0.36, p<0.05)$ and glycosylated hemoglobin $(r=0.43, p<0.01)$, but not with age $(p=0.21)$ and blood pressure $(p=0.82)$. These results are in accordance with previous evidence on the positive correlation of glycemia and HbA1c with the number of SGLT1 cotransporters [2, 6].

Norton et al. [7] point out that the increased expression of SGLT1 in patients with T2D may develop as a compensatory mechanism to increase glucose reabsorption and could therefore potentially limit the efficacy of SGLT2 inhibitors. Indeed, under physiologic conditions, the SGLT2 accounts for about $95 \%$ of filtered glucose reabsorption (160-180 g/day) in the S1 and S2 segments of the proximal tubule, while the pharmacologic treatment with SGLT2-specific inhibitor treatment reduces the glucose reabsorption by $50-70 \%$ [8]. Regarding the properties and anatomic distribution of the SGLT1 and SGLT2 cotransporters, it is also worth noting that SGLT2 is primarily expressed in the kidney, while SGLT1 does not solely mediate the glucose reabsorption of the remaining filtered glucose (about $3-5 \%$ ) in the last S3 segment of the proximal tubule [8], but it is also the main sodium/glucose cotransporter in the small gut. It has been hypothesized that SGLT1 activity could be increased as a compensatory mechanism in case of a massive tubular glucose load overwhelming the reabsorption capacity of the SGLT2 cotransporter as happens during the supra-physiologic glycosuria induced by glucose-lowering therapy with SGLT2 inhibitors [8]. However, SGLT2 selective inhibitors have been reported to be less effective in patients with moderate to severe renal dysfunction [9], which is an issue of considerable relevance considering that it affects $30-40 \%$ of all diabetic patients. In this setting, the inhibition of SLGT1 may be a promising option for achieving better glycemic control [10]. In fact, intestinal SLGT1 is responsible for most of the glucose absorption, and its blockade induces an increased glucose load to the distal gut [10]. This article does not contain any new studies with human or animal subjects performed by any of the authors.

SGLT1 mutations in mouse models may offer valid examples of SGLT1 inhibition because these phenotypes show a lack of effect of SGLT1 
in the intestinal lumen with severe osmotic diarrhea and metabolic acidosis caused by the non-absorbed excess of glucose $[11,12]$. Of note, the administrations of nonselective and selective SGLT1 inhibitors in humans are not accompanied by these serious adverse effects. Indeed, sotagliflozin, also known as LX4211, a nonselective SGLT2/1 inhibitor, was reported to be safe and well tolerated (namely without gastrointestinal side effects) when administered to 36 patients with T2D in a once-daily oral dose of 150 or $300 \mathrm{mg}$ for 28 days [13]. The main reason for its safety was ascribed to the incomplete inhibition of intestinal SGLT1. Similarly, GSK-1614235, a selective SGLT1 inhibitor, has been administered without serious safety issues to 12 healthy subjects in a 20-mg oral dose in a randomized controlled trial [14]. The plasma concentrations of GSK-1614235 indicated very low levels of the active molecule with rapid clearance and a significant $50 \%$ glucose reabsorption reduction compared to placebo. In both studies, SLGT1 inhibitor administration obtained satisfactory glycemic control, suggesting that even a low dose of SLTG1 inhibitors may be sufficient to correct hyperglycemia.

According to the potential of enhanced glycemic control through SGLT1 inhibition that also avoids the glycosuria-related side effects of SGLT2 inhibitors, particularly genitourinary tract infections, Goodwin et al. [15] recently reported the discovery of LX2761, a more potent SGLT1 inhibitor restricted to the intestinal lumen that delays intestinal glucose absorption in vivo. The authors disclosed LX2761 after the methyl substitution of the xyloside core of sotagliflozin, which guaranteed a slightly higher potency at SGLT1 and, more importantly, compatibility with synthesis and starting material availability. As expected with the delayed glucose absorption that is the prevalent mechanism of SGLT1 inhibition in the intestine, male C57 mice treated with LX2761 exhibited a significant increase in cecal glucose levels and decrease in cecal $\mathrm{pH}$ due to fermentation of glucose to short chain fatty acids in the cecum. LX2761 was designed to remain in the intestine after oral delivery to inhibit SGLT1 locally without affecting the SGLT1/2 mechanism in the kidney. LX2761 resulted as a chemically stable and very potent inhibitor against SGLT1 and SGLT2 in vitro but acted as a selective SGLT1 inhibitor in vivo in the gastrointestinal tract. This new SLGLT1 antagonist could represent a valid therapeutic strategy in the future for the treatment of diabetes, particularly in patients with renal impairment who may benefit less from the renal mechanism of selective SGLT2 inhibitors.

\section{CONCLUSION}

In conclusion, further randomized controlled trials comparing the effects of selective and nonselective SLGT1 inhibitors in patients with T2D and concomitant chronic kidney disease are warranted to assess the efficacy and the safety of these drugs according to the extant glomerular filtration rate. Indeed, further stratified analyses in other subgroups of patients with T2D are needed to better elucidate whether those presenting with higher expression levels of renal SGLT1 may be exposed to an increased risk of hypoglycemia or other complications, particularly in the context of the polypharmacy that typically characterizes patients with T2D and other comorbid conditions.

\section{ACKNOWLEDGEMENTS}

No funding or sponsorship was received for this study or publication of this article. The article processing charges were funded by the authors. All named authors meet the International Committee of Medical Journal Editors (ICMJE) criteria for authorship for this manuscript, take responsibility for the integrity of the work as a whole and have given final approval for the version to be published.

Compliance with Ethics Guidelines. This article does not contain any new studies with human or animal subjects performed by any of the authors.

Disclosures. Leonardo Spatola, Silvia Finazzi, Claudio Angelini, Marco Dauriz and Salvatore Badalamenti have nothing to disclose. 
Open Access. This article is distributed under the terms of the Creative Commons Attribution-NonCommercial 4.0 International License (http://creativecommons.org/licenses/ by-nc/4.0/), which permits any noncommercial use, distribution, and reproduction in any medium, provided you give appropriate credit to the original author(s) and the source, provide a link to the Creative Commons license, and indicate if changes were made.

\section{REFERENCES}

1. Hummel CS, Lu C, Loo DD, et al. Glucose transport by human renal $\mathrm{Na}+$ /D-glucose cotransporters SGLT1 and SGLT2. Am J Physiol Cell Physiol. 2011;300:C14-21.

2. Tahrani AA, Barnett AH, Bailey CJ. SGLT inhibitors in management of diabetes. Lancet Diabetes Endocrinol. 2013;1(2):140-51.

3. Solini A, Rossi C, Mazzanti CM, et al. Sodium-glucose co-transporter (SGLT)2 and SGLT1 renal expression in patients with type 2 diabetes. Diabetes Obes Metab. 2017;19(9):1289-94.

4. Tabatabai NM, Sharma M, Blumenthal SS, et al. Enhanced expressions of sodium-glucose cotransporters in the kidneys of diabetic Zucker rats. Diabetes Res Clin Pract. 2009;83(1):e27-30.

5. Vrhovac I, Balen Eror D, Klessen D, et al. Localizations of $\mathrm{Na}(+)$-D-glucose cotransporters SGLT1 and SGLT2 in human kidney and of SGLT1 in human small intestine, liver, lung, and heart. Pflugers Arch. 2015;467(9):1881-98.

6. Rahmoune H, Thompson PW, Ward JM, et al. Glucose transporters in human renal proximal tubular cells isolated from the urine of patients with non-insulin-dependent diabetes. Diabetes. 2005;54(12):3427-34.
7. Norton L, Shannon CE, Fourcaudot $\mathrm{M}$, et al. Sodium-glucose co-transporter (SGLT) and glucose transporter (GLUT) expression in the kidney of type 2 diabetic subjects. Diabetes Obes Metab. 2017;19(9):1322-6.

8. Liu JJ, Lee T, DeFronzo RA. Why do SGLT2 inhibitors inhibit only $30-50 \%$ of renal glucose reabsorption in humans? Diabetes. 2012;61:2199-204.

9. Yale JF, Bakris G, Cariou B, et al. Efficacy and safety of canagliflozin in subjects with type 2 diabetes and chronic kidney disease. Diabetes Obes Metab. 2013;15:463-73.

10. Gorboulev V, Schurmann A, Vallon V, et al. $\mathrm{Na}(+)$-D-glucose cotransporter SGLT1 is pivotal for intestinal glucose absorption and glucose-dependent incretin secretion. Diabetes. 2012;61:187-96.

11. Freitas HS, Anhe GF, Melo KF, et al. Na(+)-glucose transporter-2 messenger ribonucleic acid expression in kidney of diabetic rats correlates with glycemic levels: involvement of hepatocyte nuclear factor-1alpha expression and activity. Endocrinology. 2008;149:717-24.

12. Martin MG, Turk E, Lostao MP, et al. Defects in $\mathrm{Na}+$ /glucose cotransporter (SGLT1) trafficking and function cause glucose-galactose malabsorption. Nat Genet. 1996;12:216-20.

13. Zambrowicz B, Freiman J, Brown PM, et al. LX4211, a dual SGLT1/SGLT2 inhibitor, improved glycemic control in patients with type 2 diabetes in a randomized, placebo-controlled trial. Clin Pharmacol Ther. 2012;92:158-69.

14. Dobbins RL, Greenway FL, Chen L, et al. Selective sodium-dependent glucose transporter 1 inhibitors block glucose absorption and impair glucose-dependent insulinotropic peptide release. Am J Physiol Gastrointest Liver Physiol. 2015;308:G946-54.

15. Goodwin NC, Ding ZM, Harrison BA, et al. Discovery of LX2761, a Sodium-dependent glucose cotransporter 1 (SGLT1) inhibitor restricted to the intestinal lumen, for the treatment of diabetes. J Med Chem. 2017;60(2):710-21. 